\title{
Knowledge management to achieve the zero-error-goal in small batch assembly
}

\author{
G. Seliger ${ }^{1}, M$. Thie ${ }^{2}$ \\ ${ }^{1}$ IWF Institute for Machine Tools and Factory Management \\ PTZ2, Pascalstraße 8-9, 10587 Berlin, Germany \\ Tel: +49-30-314-22014 \\ Fax: +49-30-314-22759 \\ e-mail: guenther.seliger@iwf-mt.tu-berlin.de \\ ${ }^{2}$ ATB Institute for Applied Systems Technology Bremen GmbH \\ Wiener Str. 1, 28359 Bremen, Germany \\ Tel: +49-421-22092-33 \\ Fax: +49-421-22092-10 \\ e-mail:thie@atb-bremen.de
}

\begin{abstract}
This paper presents the achieved results using a new approach to identify the causes of errors and to define appropriate measures to improve processes in small batch and complex assembly. It includes a description on the procedure and tools developed for the identification of error causes, the introduction of the tools in a final assembly and the achieved benefits.
\end{abstract}

Keywords

quality, error causes, knowledge management, IT support, small batches, assembly

\section{INTRODUCTION}

The global market and the different quality requirements such as QS 9000 or VDA 6.1 force companies to ensure stable product and process quality at minimal costs. The TQM approach enables a reduction of quality costs by reducing errors through the establishment of quality capable processes. In order to reduce errors, methods and tools for the identification of error causes and for the definition of actions to improve the processes are necessary. For mass and series production 
different methods such as Statistical Process Control (SPC) are available and applied in industry. However, in small batch and complex assembly, these methods normally can not be directly applied due to the small amount of available quality data.

This paper presents, therefore, a method for the identification of quality critical processes and error causes to improve processes. The emphasis in this paper is put upon the introduction of a knowledge and model based approach in order to establish an intelligent decision support system for TQM. In addition, the results and benefits of the usage of such a system to reach the Zero-Error-Goal in final assembly are presented.

\section{KNOWLEDGE AND MODEL BASED APPROACH TO SUPPORT THE ERROR ANALYSIS}

A survey of possible approaches for the identification of error causes in small batch assembly such as

- Statistical Approaches (Al-Salti, 1994),

- System and Model Based Approaches (Isermann, 1996),

- System and Knowledge Based Approaches (Isermann 1998) and

- Learning Approaches (Sorsa, 1993)

shows that each one has certain advantages but also some disadvantages. The main problem related to small batches is that the sample size may not be sufficient to fulfil the requirements regarding statistical significance. The basic approach for the identification of critical processes of the error that occur in assembly is to apply a combination of statistical methods and knowledge based systems to interpret information (Thie, 1998). Additionally the modelling approach is used to support the classification of information and the learning approach is applied to update the 'rules'. The main problems applying of such an approach are as follows: definition of a consistent and reliable rule set, to extend these rules to new error types and situations which have not appeared before etc. This paper concentrates on the application of such a method in an industrial environment.

The procedure for the identification of critical processes and error causes starts with classifying errors in such a way that each error in a group has similar classes of critical processes and error causes (Figure 8). Between the error, critical processes and error causes, 'a priori'-rules are defined in the following way 'process may cause a deviation in a deviation class with a specific probability'. Since it is obviously not possible to define rules valid for all possible cases, additional information on the processes, products and statistical analysis are used to increase or decrease the 'a priori'-probabilities of the critical processes / error causes. More information about the developed method can be find in Thie, 1998 or Wangberg, 1998. 


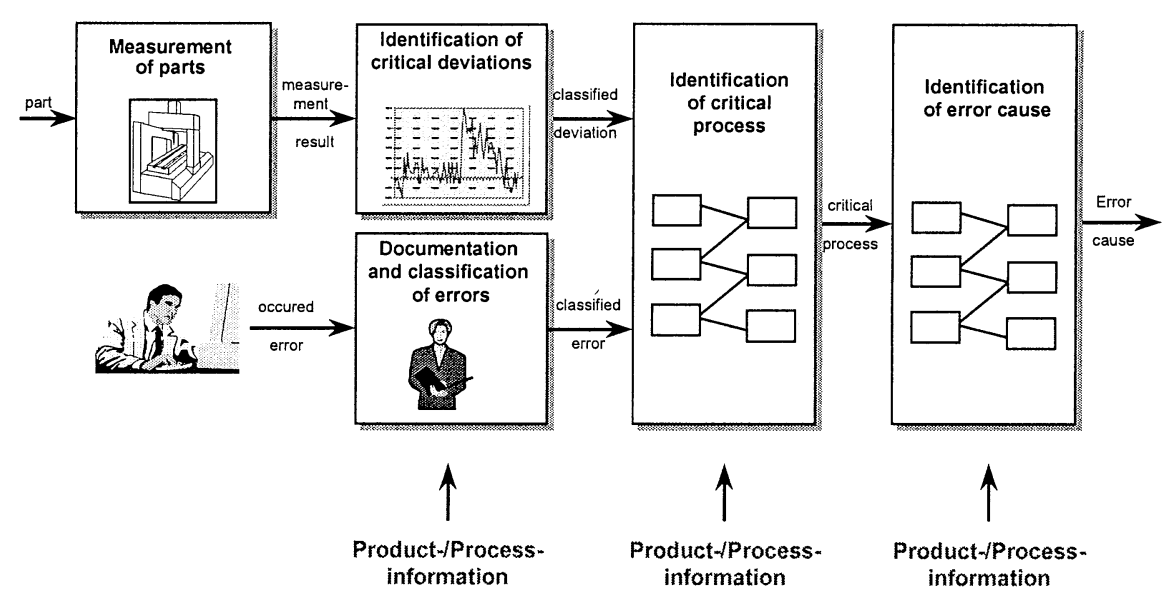

Figure 8: Procedure for the identification of error causes [Thie, 98]

\section{USAGE OF KNOWLEGDE BASED METHODS AND TOOLS IN A FINAL ASSEMBLY OF AIRCRAFTS}

\subsection{Introduction of the systems in the final assembly}

The developed knowledge based methods and tools are used as one example to support the quality improvement of a final assembly of aircraft's. One problem in this application area is the assembly of pipes for the hydraulic systems of the aircraft.

In the past several problems occurred w.r.t. the final assembly of pipes. Pipes could not be assembled and extensive actions were necessary to solve the problems. This caused high additional efforts and costs to identify the causes of the quality problems and to define actions to improve the process and product quality. However, in order to identify the true cause and to define appropriate actions, comprehensive knowledge along the entire production process about the processand product-relation and their relation to errors and error causes in a structured form is necessary. So the knowledge based method is introduced in this area to support process improvements with minimal efforts. By this, stable processes with less assembly problems should be achieved.

The main task during the introduction of such an approach (tool supported) in a specific area constitutes besides the organisational and technical integration, the capturing of useful quality, product and process data, and the definition of the way in which to build up the initial knowledge base to identify error causes and to define actions to improve process and product quality. Several steps to introduce such methods have to be performed: 
1.) Definition of knowledge base

Before the methods can be applied, in a first step the knowledge base with the error/process/cause/action-relations has to be defined for the application area 'final assembly'. The initial version of the knowledge base is specified using the experience of responsible people from earlier error analysis at final assembly, in quality assurance, production of pipes and design \& planning. Using the learning part in the method the knowledge base will be later updated step by step.

2.) Organisational integration

The procedures to use the methods have to be to specified. Based on the drawings, work orders and other documents from the design \& planning the pipes are assembled. If it is not possible to assemble a part as required, a solution is sought by the operator or in more complex problems by production or quality engineers to solve the actual problem. These errors will be documented in a quality documentation system which is available at all assembly stations (Figure 9). From time to time the error documentation is analysed to identify critical errors, i.e. reoccurring errors or important errors e.g. due to high costs to solve the error. For these errors the identification of error causes will be done.

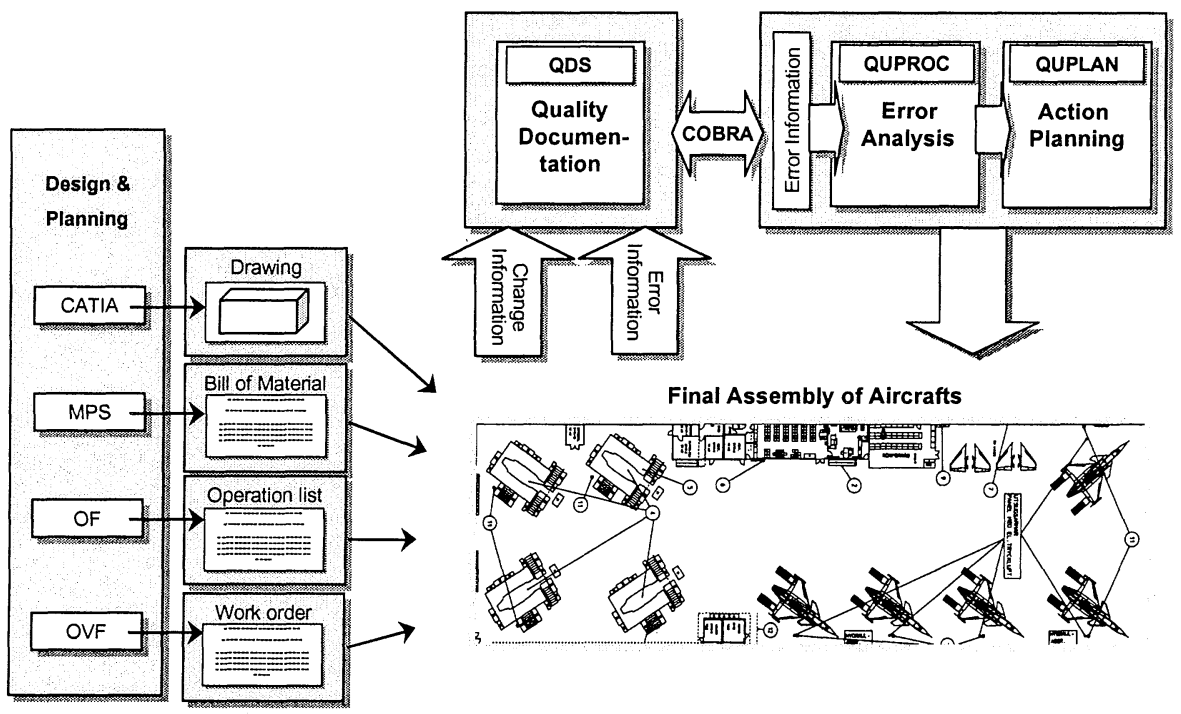

Figure 9: Organisational integration of QUPROC and QUPLAN (QUETA, 1999)

Based on the error type the analysis is started in a specific assembly area (final or fuselage assembly, pipe production etc.). First the critical process has to be identified. Necessary information to evaluate the knowledge base is collected from other systems. However, if some required information is not available in other systems, and, therefore, cannot be automatically obtained, an operator has to provide this information. In a similar way the error cause and actions to improve the process are identified. 
3.) Technical integration

Due to the large amount of necessary quality, process and product data the software tools QUPROC (identification of quality critical processes) and QUPLAN (definition of action plans to improve the processes) have been developed. The tools require a large amount of information which is normally available in other legacy systems of the company (PDM, PPC etc.). To get a part of this required information automatically, the developed tools have to be technically integrated into these systems of the company. The following information has to be transferred from different systems (e.g. PPC, QDS, BDE etc.) to use the tools in the presented application area 'final assembly':

- Process information (information on processes within the production of aircraft's),

- Product information (information on parts, subassemblies or the aircraft),

- Error information (information on the error and on the faulty product),

- Change information (information on product and process changes).

The systems which have to be integrated are using different hardware and operating systems. To support the integration, middleware technology CORBA is used to get data from different legacy systems and provide the functionality in a web browser at different places in the company (Figure 10).

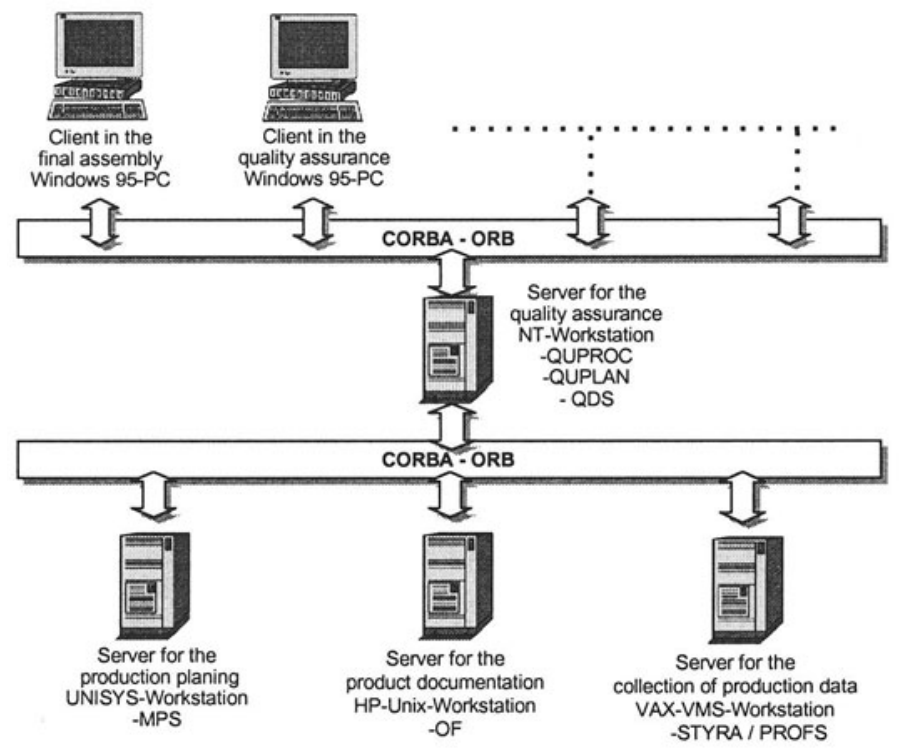

Figure 10: Technical integration of QUPROC and QUPLAN in final assembly

\subsection{Achieved results}

To better understand the benefits of such a method for process improvement the usage of the support tools will be described through a real example in the following. 
The starting point for an analysis is the description of an occurred error. In the example considered, a pipe can not be assembled. The following processes could be responsible for this error:

- Design \& Planning: The given Jocuments (drawings, working instructions) for the pipe or related parts have an error.

- Production of Pipes: The pipe did not reach the target, since an error occurred in the bending of the pipe.

- Fuselage assembly: The fuselage did not reach the target, since the fuselage assembly was not executed in the right way.

- Final assembly: The error occurred in final assembly since other parts were not assembled as defined in the working instructions.

Accordingly QUPROC calculates based on knowledge of the error/processrelations the 'a priori'-probabilities for the critical processes (Figure 11).

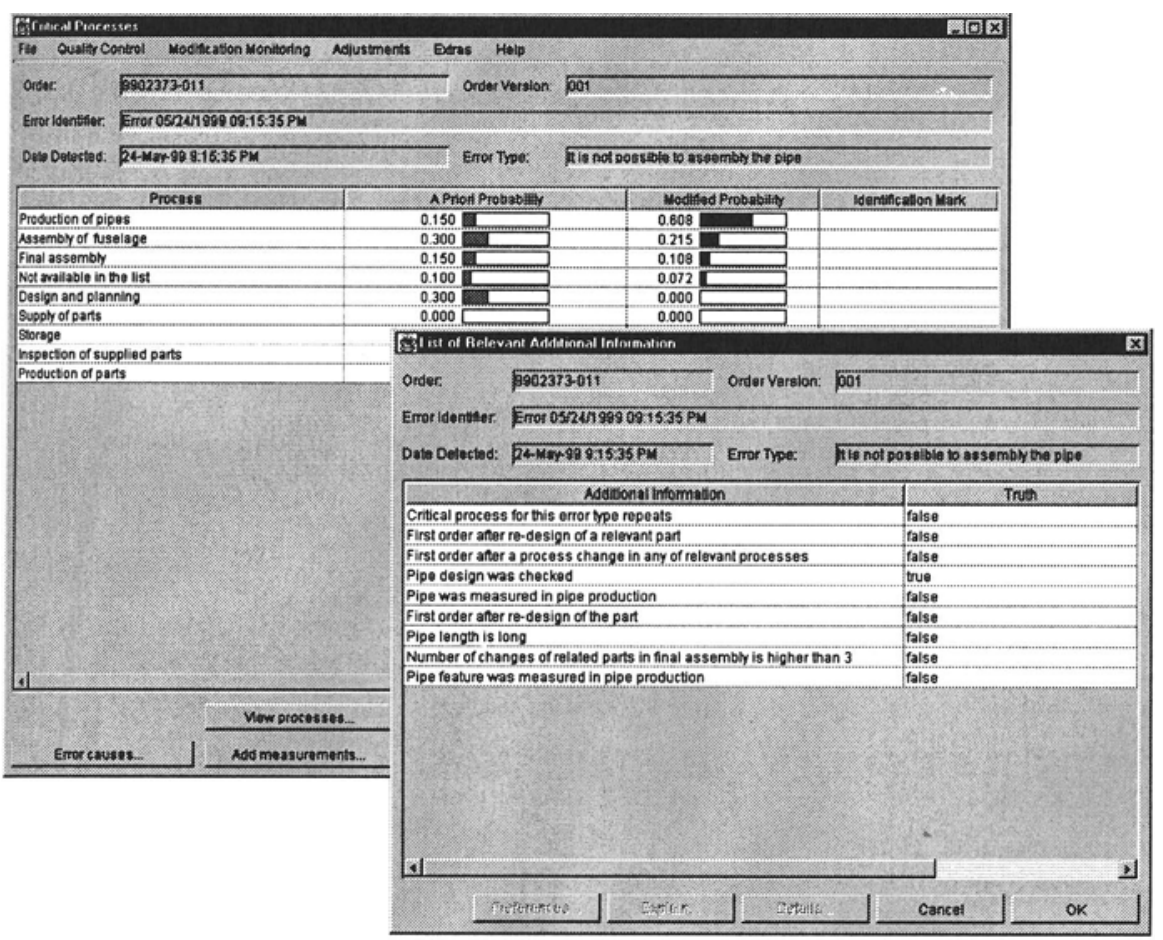

Figure 11: Identification of the critical process for the occurred assembly error

Since it is not possible to identify the critical process with only the 'a priori'probabilities, additional information is used in modification rules to update the probabilities for the concrete error situation. For the actual error the following information is relevant:

- Verification if the given documents for the faulty or related part were changed recently. 
- Verification if the faulty pipe was inspected after the bending process.

- Verification if small changes were made to a related part in final assembly. Since this could have influences to the pipe.

The necessary information can be found in the different legacy systems of the company. Using the implemented interfaces and internal functionality QUPROC is able to evaluate the necessary information automatically. Based on the application of the rule base, the probabilities for the critical processes calculated that the responsible process can be identified with a sufficient probability (Figure 11).

Once the critical process 'Production of Pipes' is identified, the list of possible error causes could be drastically reduced. In a similar way the error cause will be identified and the action to improve the process will be defined.

The presented analysis of an occurred error in final assembly shows that for such complex cases a lot of different information and knowledge is necessary. Such information and knowledge is normally distributed over different information systems and people in a company and since specific errors are occurring quite rarely, support of such systems with the corporate knowledge are effective for identification of error causes. The following benefits could be achieved using the tools QUPROC and QUPLAN for process improvement in the final assembly of aircraft's:

- Reduction of errors in final assembly by identification of the true causes for errors,

- Reduction of efforts and time for the identification of reasons for errors by the availability of error knowledge,

- Less dependency on employees with high experience by enabling less experienced employees to analyse errors,

- Higher transparency in the analysis of error causes by defined and repeatable procedures,

- Reduction of efforts and time to reach a stable assembly process after changes by the availability of error knowledge on previous changes,

- Preventive avoidance of errors by usage of the quality knowledge base.

The verification and testing of the tools is in progress and measurable benefits will be provided in QUETA, 1999.

\section{CONCULSION}

The methods and tools to support process improvement in small batch assembly using a combination of knowledge and model based, statistical and learning approaches presented in this paper have proven their efficiency to support this task. To further optimise such tools and their introduction into an industrial environment, future research should concentrate on the increased usage of obtained knowledge for the planning process, on the standardisation of interfaces to legacy systems and on the transfer of such principals to serial manufacturing and assembly. 


\section{ACKNOWLEDGEMENTS}

The work here described is supported by the European Community, through the ESPRIT project QUETA.

\section{REFERENCES}

Al-Salti, M.; Statham, A. (1994): A Review of the Literature on the Use of SPC in Batch Production, Quality and Reliability Engineering International, 1, 49-61

Isermann, R. (1996): Modellgestützte Überwachung und Fehlerdiagnose Technischer Systeme (Part 1), Automatisierungstechnische Praxis, 5, 9-20

Isermann, R. (1998): Wissensbasierte Fehlerdiagnose technischer Prozesse, Automatisierungstechnik, 11, 421-426

QUETA (1999) Field-Test and Evaluation: Results of testing and evaluation of tools. Planed Public Report of QUETA project, No. 22367, ESPRIT Domain 8 Task 8.5.

Sorsa, T.; Koivo, H. N. (1993): Application of Artificial Neural Networks in Process Fault Diagnosis, Automatica, 4, 843-849

Thie, M; Stokic, D. (1998): Knowledge Based Methods and Tools for TQM in Small Batch Flexible Manufacturing and Complex Assembly, in: Intelligent Systems for Manufacturing: Multi-Agent Systems and Virtual Organizations, (Eds. Luis M. Camarinha-Matos et al.) Kluwer Academic Publishers, Boston, Dordrecht, London, 459-468

Wangberg, K.-G. et al. (1998): IT based Quality Engineering Tools for Assembly and Small Batches Manufacturing, in: Changing the Ways We Work - Shaping the ICT-solutions for the Next Century (Eds. N. Martensson et al.), ISO Press, Amsterdam, Berlin, Oxford, Tokyo, Washington, 141-153

\section{BIOGRAPHY}

Prof. Dr.-Ing. Günther Seliger born 1947, studied economic engineering at the Technical University of Berlin and accomplished his PhD by Prof. Spur. After different leading positions at the Institute for Machine Tools and Factory Management (IWF) of the TU Berlin as well as at the Fraunhofer Institute for Production Systems and Design Technology (IPK-Berlin), he became in October 1988 a Professor for assembly technology at the IWF of the TU Berlin. From April 1997 to March 1999 he was Vicepresident of the TU Berlin.

Dipl.-Ing. Michael Thie born 1968, studied Mechanical Engineering at the University of Hanover. Since 1993 he has been working at ATB Institute for Applied System Technology Bremen $\mathrm{GmbH}$. His key areas of activity are quality management, quality methods and techniques, process optimisation and introduction of IT-Systems. Since 1998 he is responsible for the area quality management. 